Supporting Information

\title{
The Mechanism of Surface-Radical Generation and Amorphisation of Crystalline Quartz Sand upon Mechanochemical Grinding
}

Kapish Gobindlal $^{1,2}$, Zoran Zujovic ${ }^{2}$, Pooja Yadav ${ }^{2}$, Jonathan Sperry ${ }^{1,2}$, \& Cameron C. Weber*1,2,3

${ }^{1}$ Centre for Green Chemical Science, University of Auckland, 23 Symonds Street, Auckland 1010, New Zealand.

${ }^{2}$ School of Chemical Sciences, University of Auckland, 23 Symonds Street, Auckland 1010, New Zealand.

${ }^{3}$ The MacDiarmid Institute for Advanced Materials and Nanotechnology, PO Box 600, Wellington 6140, New Zealand.

\section{Contents}

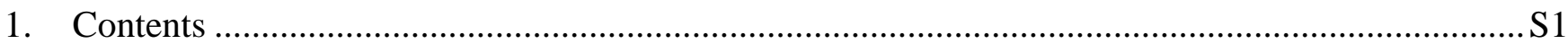

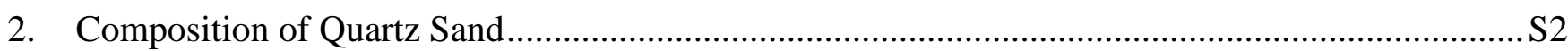

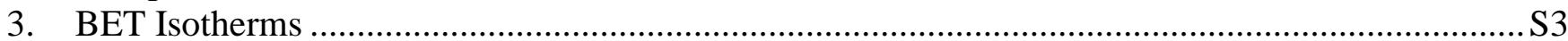

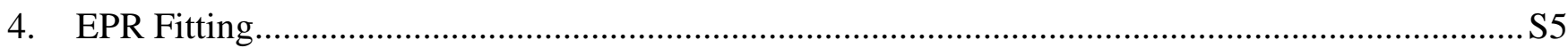

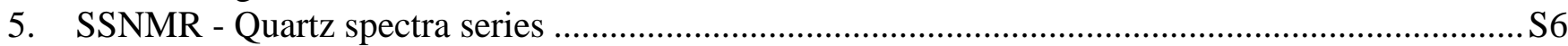

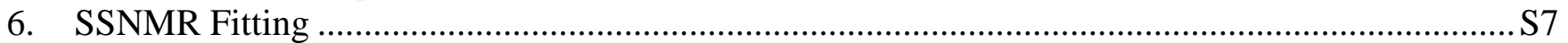




\section{Composition of Quartz Sand}

Table S1. X-Ray fluorescence of as-received quartz sand.

\begin{tabular}{|l|c|}
\hline & $\%$ \\
\hline $\mathrm{SiO}_{2}$ & 99.2 \\
\hline $\mathrm{Al}_{2} \mathrm{O}_{3}$ & 0.04 \\
\hline $\mathrm{Fe}_{2} \mathrm{O}_{3}$ & 0.06 \\
\hline $\mathrm{CaO}$ & 0.02 \\
\hline $\mathrm{MgO}$ & $<0.01$ \\
\hline $\mathrm{SO}_{3}$ & 0.03 \\
\hline $\mathrm{K}_{2} \mathrm{O}$ & 0.02 \\
\hline $\mathrm{Na}_{2} \mathrm{O}$ & $<0.01$ \\
\hline $\mathrm{MnO}_{2}$ & $<0.01$ \\
\hline $\mathrm{TiO}_{2}$ & 0.01 \\
\hline $\mathrm{P}_{2} \mathrm{O}_{5}$ & 0.01 \\
\hline $\mathrm{H}_{2} \mathrm{O}$ & $<0.01$ \\
\hline $\mathrm{Loss}_{2}$ on Ignition & 0.10 \\
\hline
\end{tabular}

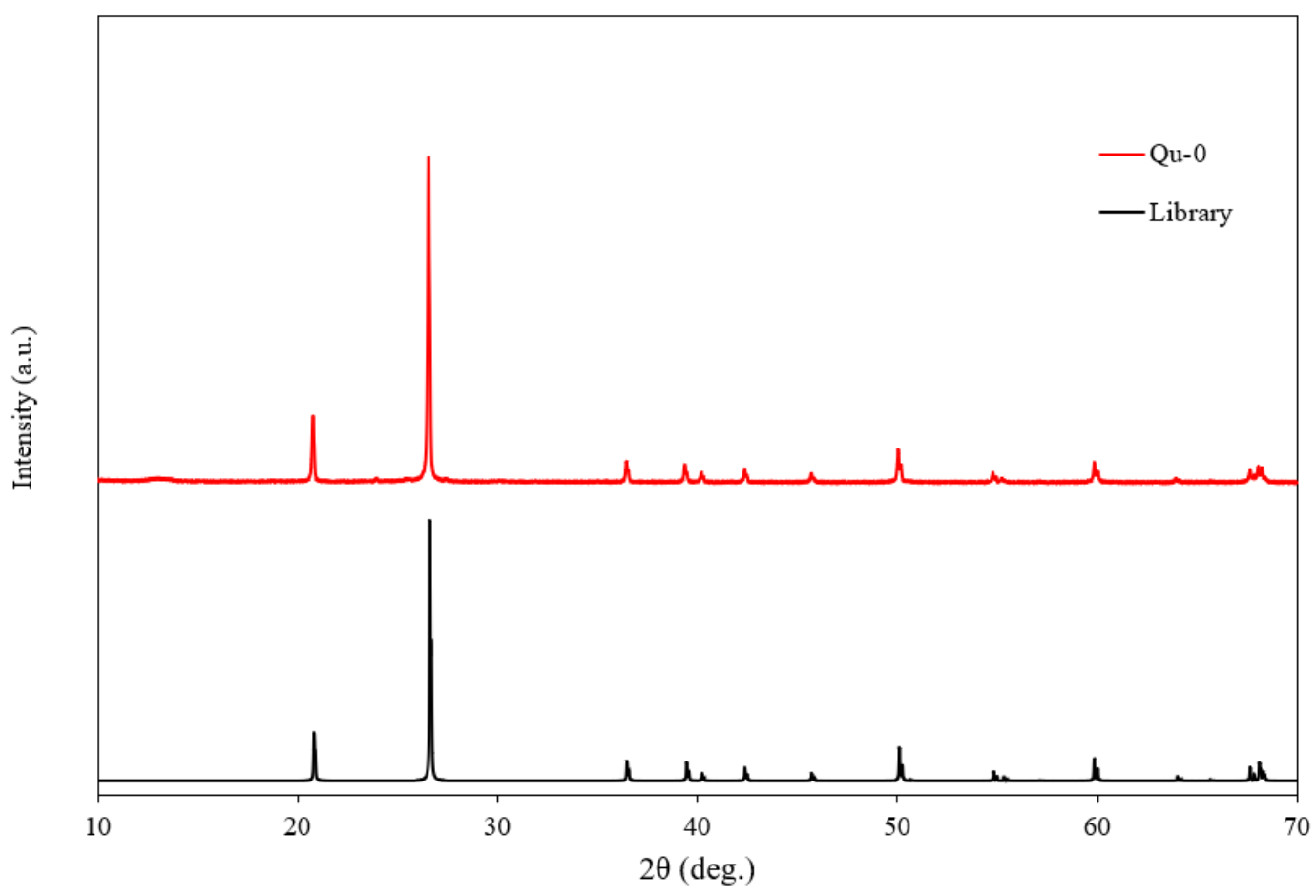

Figure S1. XRD diffraction pattern of the quartz sand starting material (red) and the library diffraction pattern for quartz as comparison. 


\section{BET Isotherms}

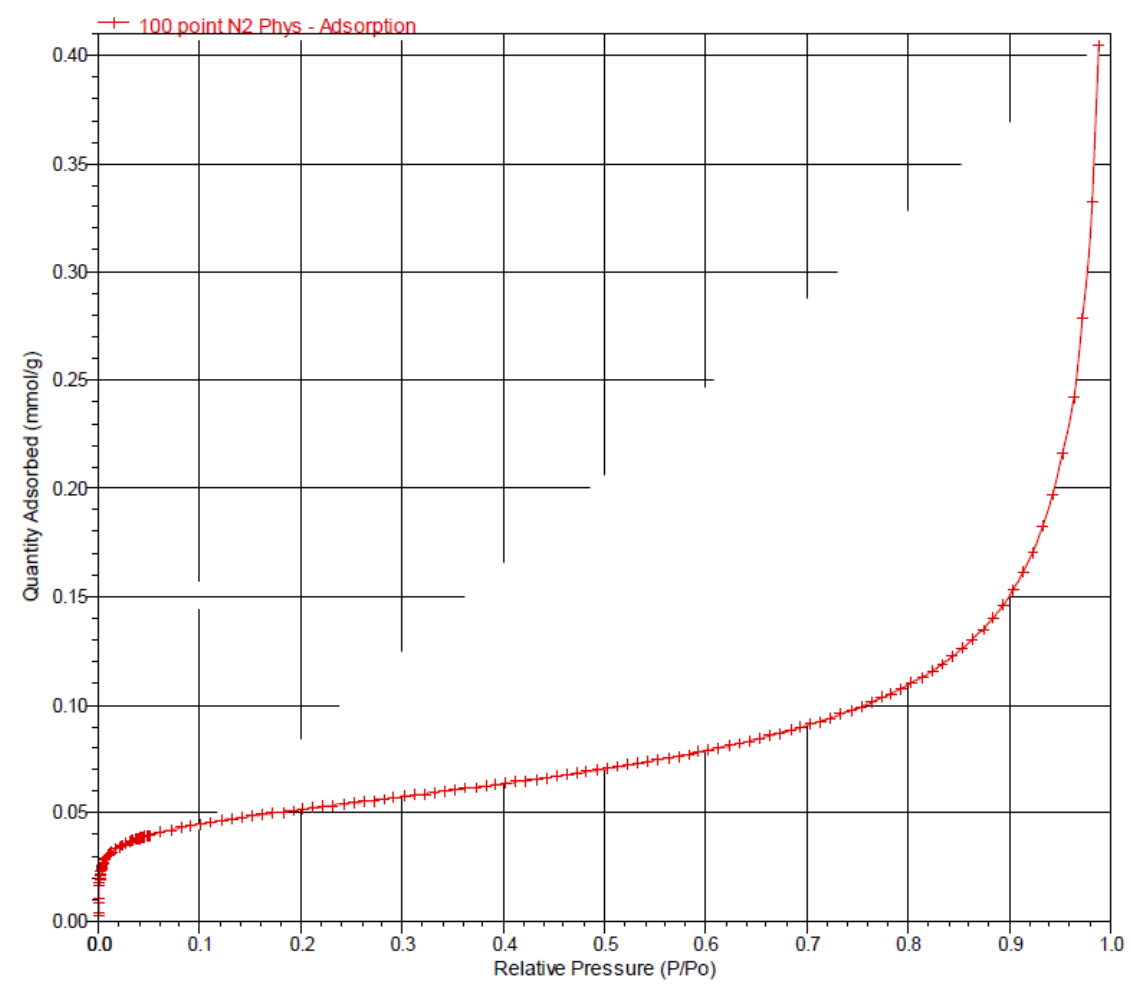

Figure S2. Qu-60 isotherm linear plot.

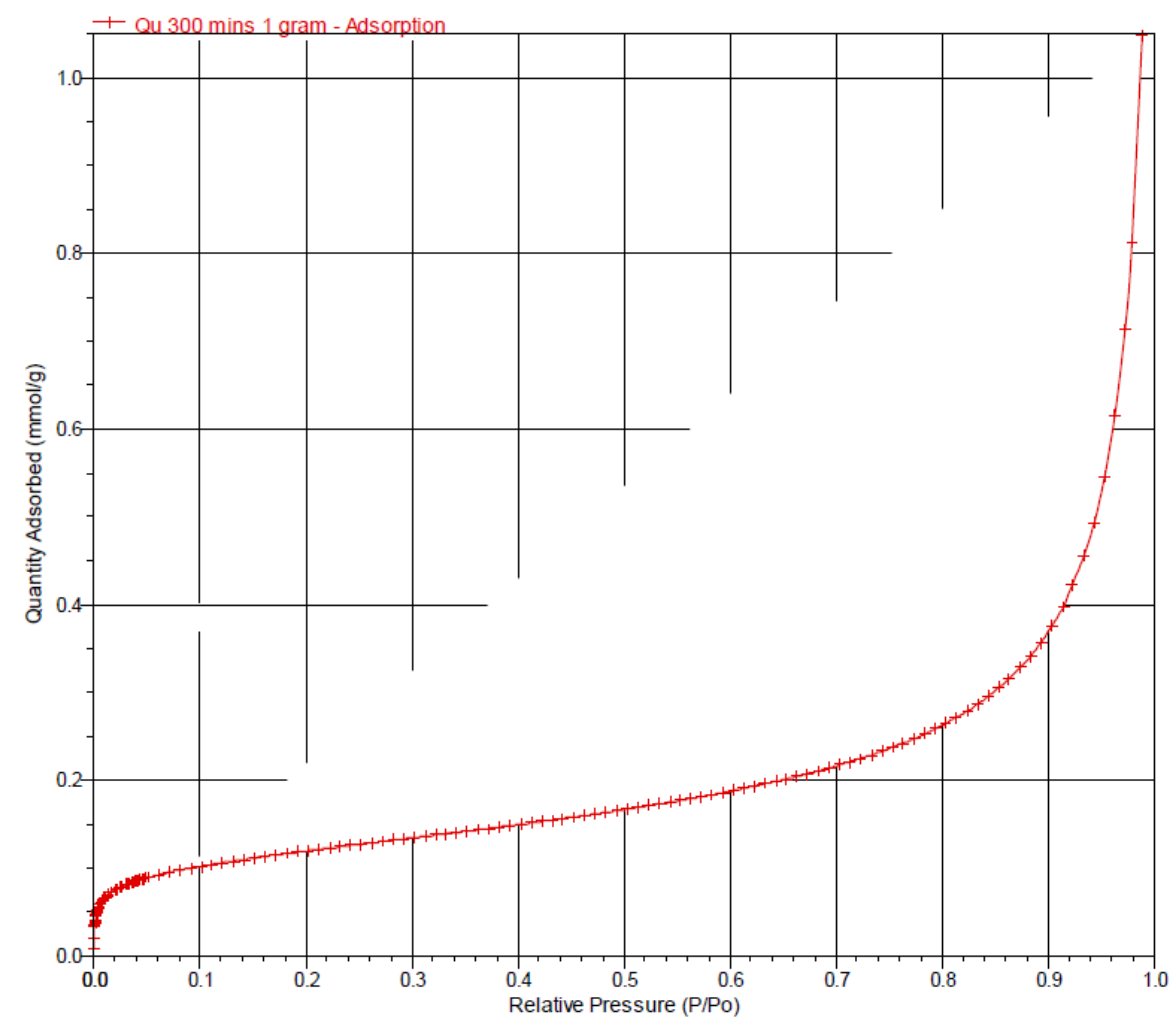

Figure S3. Qu-300 isotherm linear plot. 


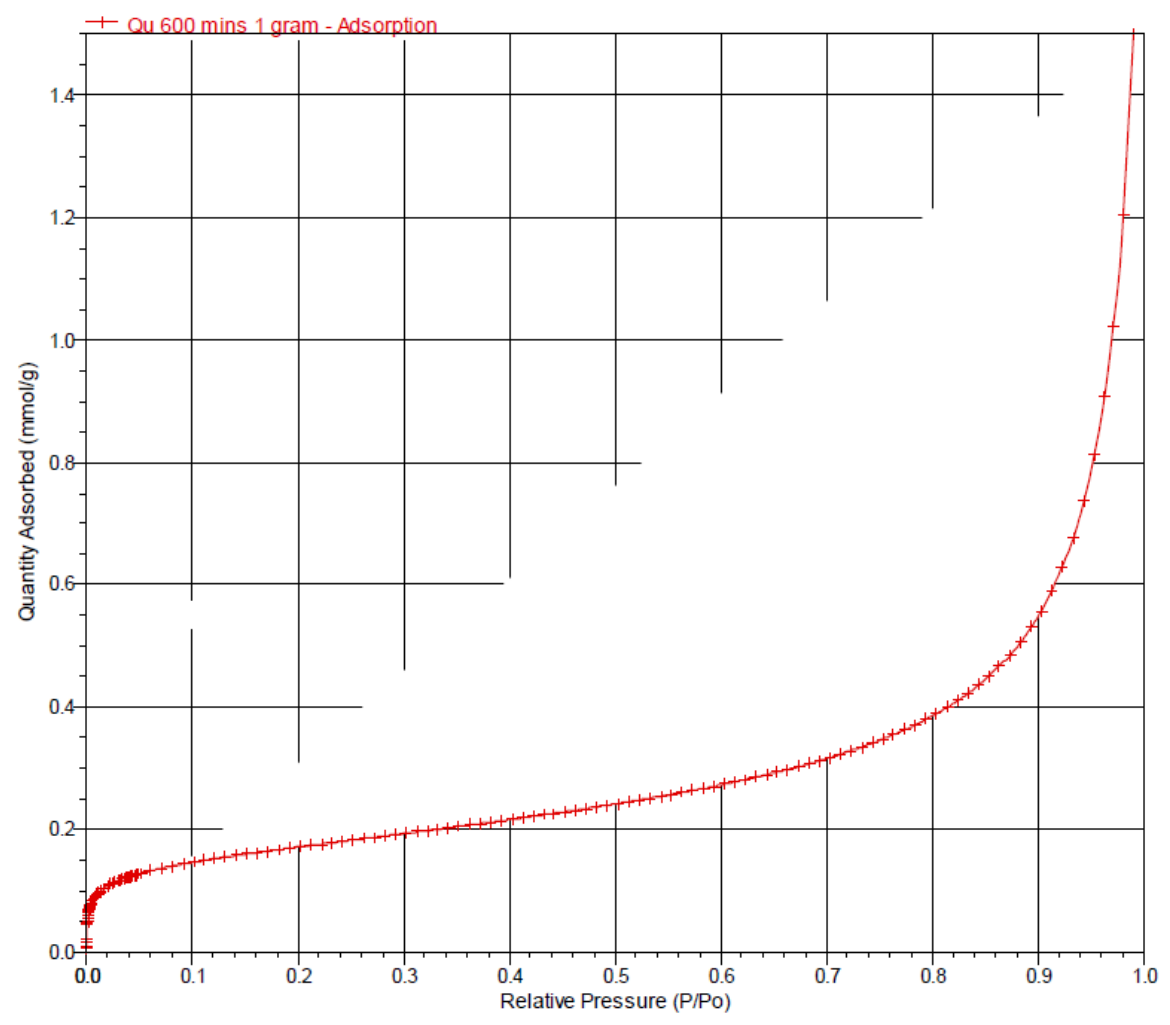

Figure S4. Qu-600 isotherm linear plot. 


\section{EPR Fitting}

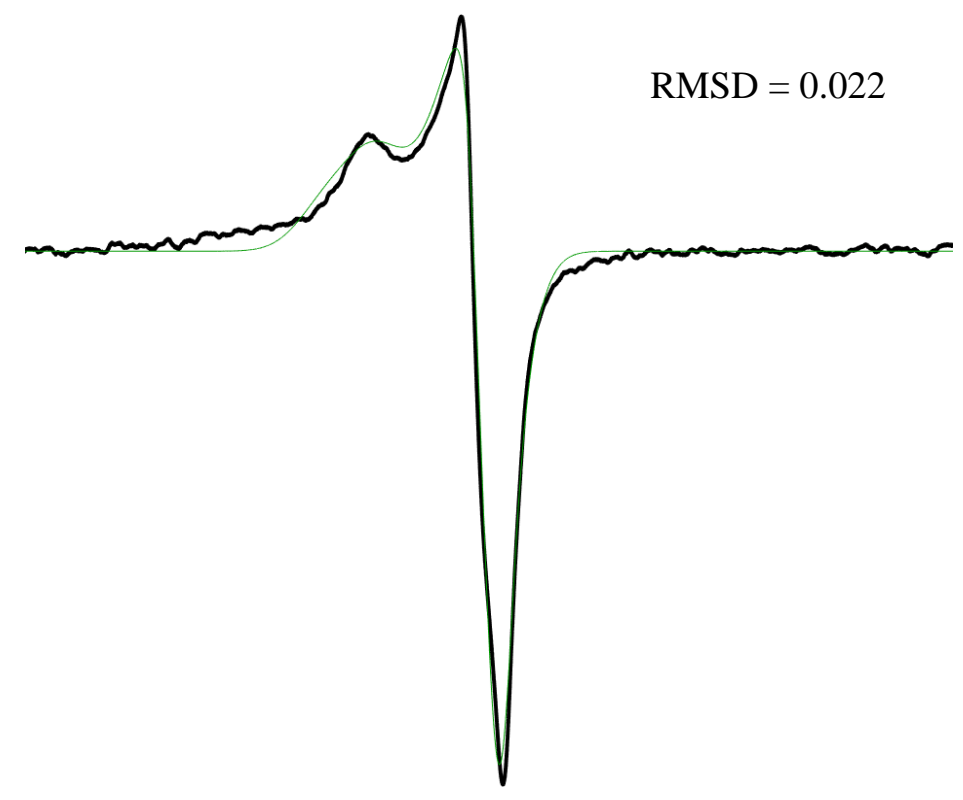

Figure S5. Fitted EPR spectrum of Qu-600. Experimental values in black and simulation in green.

Table S2. EPR fitting parameters using EasySpin Software.

\begin{tabular}{|l|c|}
\hline & Qu-600 \\
\hline \# of spins & ${ }^{1 / 2}$ \\
\hline Nucleus & ${ }^{29} \mathrm{Si}$ \\
\hline $\mathrm{g}_{1}$ & 2.0009 \\
\hline $\mathrm{g}_{2}$ & 2.0005 \\
\hline $\mathrm{g}_{3}$ & 2.0284 \\
\hline $\mathrm{A}_{1}$ & 15 \\
\hline $\mathrm{A}_{2}$ & 11 \\
\hline $\mathrm{A} 3$ & 42 \\
\hline
\end{tabular}




\section{SSNMR - Quartz spectra series}
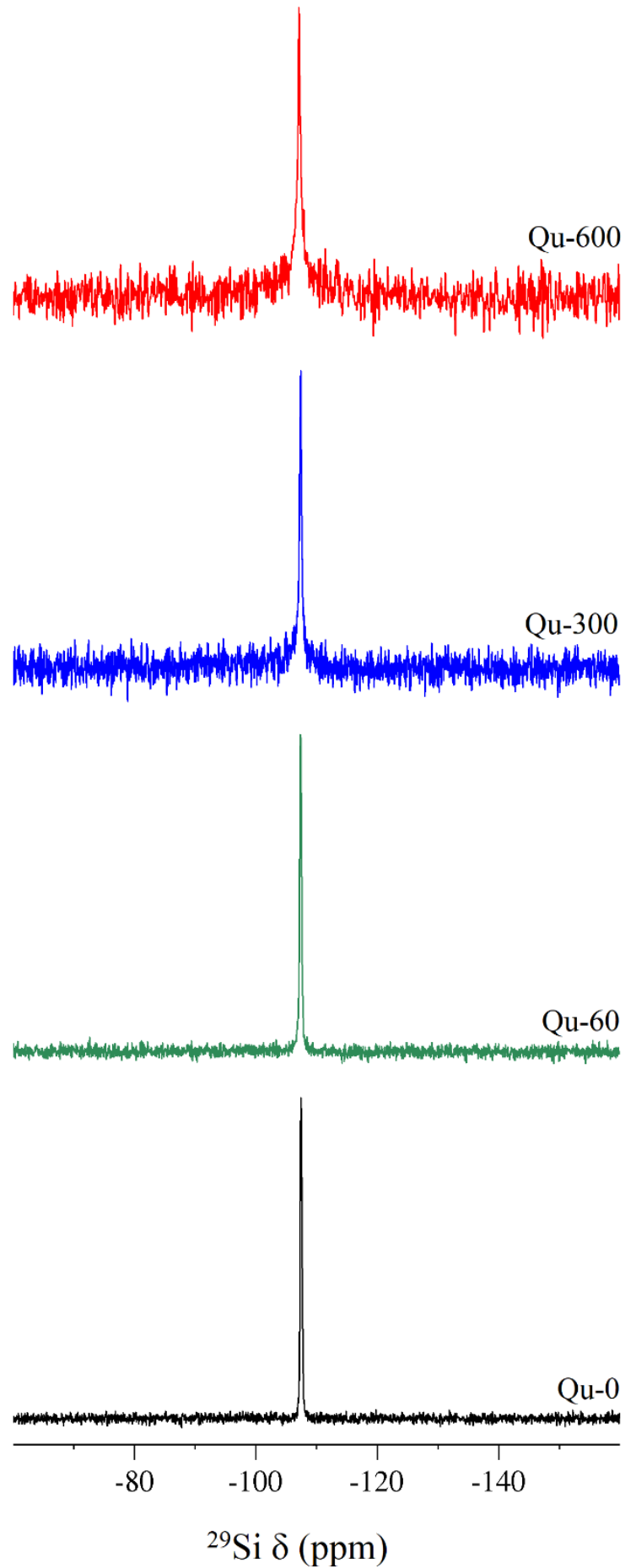

Figure S6. ${ }^{29}$ Si SSNMR spectra of quartz sand samples. No line broadening. 


\section{SSNMR Fitting}

Table S3. SSNMR fitting parameters for amorphous components using Bruker Software.

\begin{tabular}{|l|c|c|c|}
\hline & Site 1 & Site 2 & Site 3 \\
\hline Intensity & 886352.1 & 5773753.6 & 8467517.7 \\
\hline $\begin{array}{l}\text { Chemical Shift } \\
(\mathrm{ppm})\end{array}$ & -91.6 & -101.0 & -109.7 \\
\hline $\begin{array}{l}\text { Line Broadening } \\
(\mathrm{Hz})\end{array}$ & 416.2 & 766.4 & 604.5 \\
\hline $\begin{array}{l}\text { Gaussian / } \\
\text { Lorentzian }\end{array}$ & 0.5 & 0.5 & 0.5 \\
\hline Area & 263750118.9 & 150168053.1 & 651602943.5 \\
\hline
\end{tabular}

Table S4. SSNMR fitting parameters for amorphous components using Bruker Software, including the partially relaxed crystalline component

\begin{tabular}{|l|c|c|c|c|}
\hline & Site 1 & Site 2 & Site 3 & Site 4 \\
\hline Intensity & 966335.5 & 5706762.1 & 4805287.3 & 7707192.8 \\
\hline $\begin{array}{l}\text { Chemical Shift } \\
(\mathrm{ppm})\end{array}$ & -91.6 & -101.0 & -107.6 & -109.7 \\
\hline $\begin{array}{l}\text { Line Broadening } \\
(\mathrm{Hz})\end{array}$ & 438.6 & 747.9 & 100.0 & 637.7 \\
\hline $\begin{array}{l}\text { Gaussian / } \\
\text { Lorentzian }\end{array}$ & 0.5 & 0.5 & 0.5 & 0.5 \\
\hline Area & 302958989.5 & 3039123628.6 & 344986226.0 & 3504492540.1 \\
\hline
\end{tabular}

